

http://www.diva-portal.org

This is the published version of a paper published in British Journal of Dermatology.

Citation for the original published paper (version of record):

Schmitt-Egenolf, M. (2016)

Physical activity and lifestyle improvement in the management of psoriasis.

British Journal of Dermatology, 175(3): 452-453

http://dx.doi.org/10.1111/bjd.14899

Access to the published version may require subscription.

N.B. When citing this work, cite the original published paper.

Permanent link to this version:

http://urn.kb.se/resolve?urn=urn:nbn:se:umu:diva-126984 


\section{Physical activity and lifestyle improvement in the management of psoriasis}

DOI: $10.1111 /$ bjd.14899

In this issue of the British Journal of Dermatology, Anna Chisholm et al. ${ }^{1}$ from Manchester present their multidisciplinary work: 'Motivational interviewing-based training enhances clinicians' skills and knowledge in psoriasis: findings from the Pso Well ${ }^{\circledR}$ study'. This study reminds us of the importance of a holistic approach to psoriasis care. It adds to a growing body of evidence that suggests we need to redefine our way of managing psoriasis, but it can also be seen as a reminder that we have to constantly develop our profession. Lifestyle management has become a major medical issue in the 21 st century. This is altogether fitting and proper; according to the World Health Organization, insufficient physical activity is a key risk factor for cardiovascular diseases, cancer and diabetes. ${ }^{2}$ Furthermore, worldwide obesity has more than doubled since 1980, and most of the world's population live in countries where overweight and obesity kills more people than underweight. ${ }^{3}$

Patients with moderate to severe psoriasis are especially vulnerable to suboptimal lifestyles. This is due to two facts: (i) as they have an elevated risk of both cardiovascular and metabolic disease, a suboptimal lifestyle is particularly dangerous; and (ii) a suboptimal lifestyle has a negative impact on psoriasis itself. However, as Chisholm et al. ${ }^{1}$ state, dermatologists still too often choose to view psoriasis as an isolated skin condition rather than a complex condition requiring discussion of lifestyle choices. This is not surprising, given the secondary socialization most of us went through during specialization. We just tend to feel more comfortable in the role of an oldstyle skin specialist, compared with the more holistic role required in contemporary clinical practice. This may also explain why many dermatologists implicitly accept the idea that biologics are the right tool to prevent comorbidities, instead of discussing required lifestyle changes. It may also explain why Chisholm et al. ${ }^{1}$ noted that 'following training, clinicians' knowledge of psoriasis-related comorbidities did not increase, nor were they more likely to explain to patients how psoriasis and behavioural factors are associated'. However, as the Swedish experience shows, those obstacles can be overcome. A decade ago the Swedish Psoriasis Registry (PsoReg) introduced measurements required for holistic psoriasis management into dermatological clinical practice, such as body mass index (BMI), waist circumference, alcohol/tobacco consumption and quality of life measurements. ${ }^{4}$ This was not initially appreciated by all dermatologists. But today, many colleagues express gratitude to those tools in PsoReg, as they encouraged them to leave their comfort zone of old-style dermatologist behaviour. After a period of adaptation, many felt empowered by these measurements to lead their patients beyond pharmaceutical treatment.

Dermatologists are often a central caregiver for patients with moderate to severe psoriasis. While technical development has created a distance between the physician and the patient in other specialties, we dermatologists still interact directly with

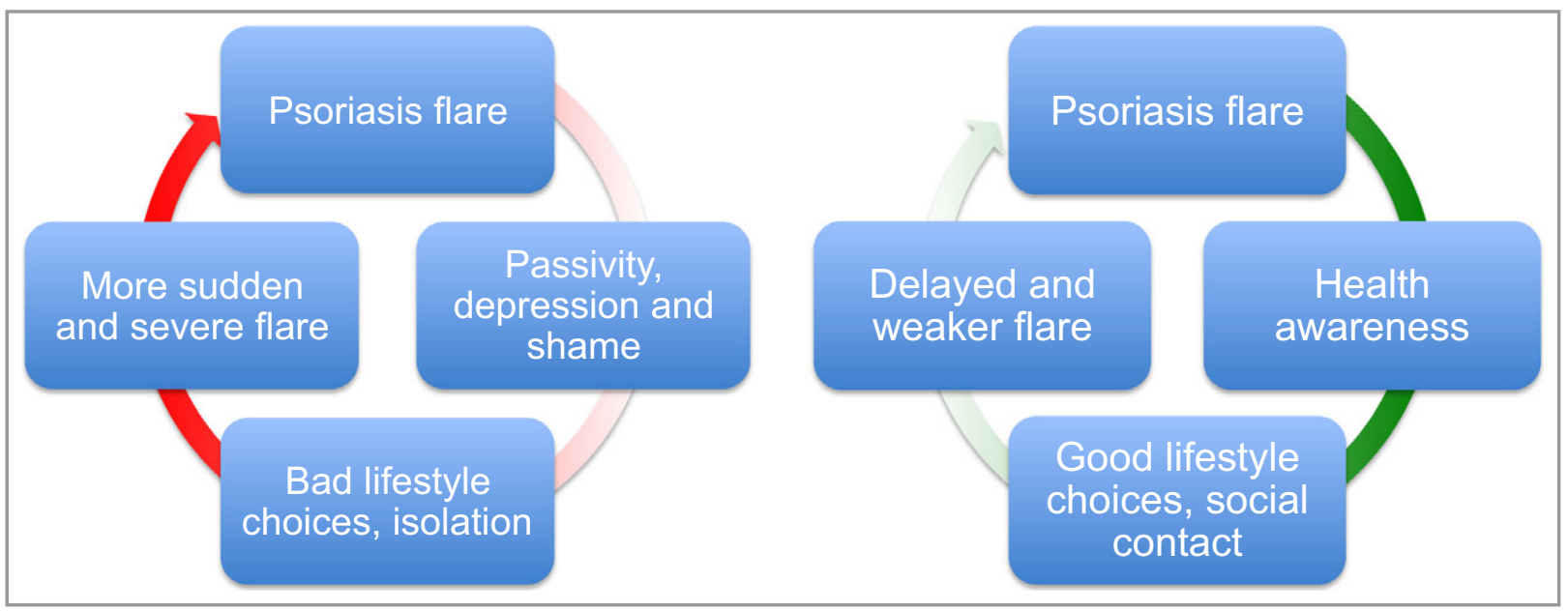

Fig 1. A flare can start either a vicious or a virtuous circle. Psoriasis flare-ups can lead to depression and despair and hence an impaired lifestyle with increased sedentary behaviour, smoking and alcohol consumption as a result (left). As physical activity affects both body and mind, it can serve as an excellent vehicle to transform this vicious circle to a virtuous one (right). 
our patients. In this encounter an excellent opportunity arises to raise and discuss health challenges such as the cardiovascular and metabolic disease risk associated with moderate to severe psoriasis; by using this dialogue to understand how the individual looks at the risks, dermatologists can initiate discussions about lifestyle improvements. Is it really OK to address lifestyle questions, one might ask? Of course it is! To withhold this information would be unethical. Once the patient is informed, we have to show avenues for the demanding journey of lifestyle improvements, an important but challenging task. ${ }^{5}$ This has to be done in an interdisciplinary way, but it is important that we dermatologists take it as our responsibility to initiate this process. We should not just rely on our colleagues in primary care in this question. A recent study by the Manchester group found that primary-care physicians did not communicate cardiovascular disease risk to patients with psoriasis in the majority of cases. ${ }^{6}$ Interdisciplinary teams supporting patients with psoriasis in this difficult journey have now been established in many dermatology clinics.

Lifestyle improvement for patients with psoriasis may involve several areas such as diet, smoking, alcohol and relaxation techniques. However, physical activity appears to be a good starting point, given the apparent positive influence on psoriasis itself alongside the potential cardiovascular and metabolic comorbidities associated with psoriasis. Furthermore, the positive influence of physical activity on psychological wellbeing can make a big difference in a patient's life. Clinical experience shows that many patients with moderate to severe psoriasis may end up in a vicious circle (Fig. 1), which may possibly be broken by physical activity.

Research on the effects of physical activity on psoriasis is a growing but still limited area. However, it already demonstrates the importance of physical activity for patients with moderate to severe psoriasis. A Portuguese study found that patients with severe psoriasis had reduced levels of physical activity compared with patients without psoriasis. ${ }^{7}$ This is not surprising, given the known barriers to physical activity experienced by patients with psoriasis. ${ }^{8,9}$ A prospective cohort study with female nurses showed that physical activity of moderate to high intensity was associated with lower risk for psoriasis, even after adjusting for BMI. ${ }^{10}$ In an article published in the BJD in 2014, Naldi et al. ${ }^{11}$ describe a randomized controlled trial over 20 weeks with overweight or obese patients with moderate-to-severe psoriasis. The active intervention combined a dietary plan with physical exercise for weight loss three times a week for at least $40 \mathrm{~min}$. The intention-totreat analysis $(n=151)$ showed an encouraging median Psoriasis Area and Severity Index reduction of $48 \%$.

As dermatologists, we have to engage ourselves in the lifestyles of our patients with moderate to severe psoriasis, not because it is easy, as the study of Chisholm et al. shows, but because it is of vital importance.

\section{Funding sources}

No external funding.

\section{Conflicts of interest}

None declared.

Department of Public Health and Clinical

M. Schmitt-Egenolf

Medicine, Dermatology, Umea University,

SE-901 87 Umea, Sweden

E-mail: marcus.schmitt-egenolf@umu.se

\section{References}

1 Chisholm A, Nelson PA, Pearce CJ et al. Motivational interviewingbased training enhances clinicians' skills and knowledge in psoriasis: findings from the Pso Well ${ }^{\circledR}$ study. Br J Dermatol 2016; doi: 10.1111 /bjd. 14837 .

2 World Health Organization. Fact sheet: Physical activity, June 2016. http://www.who.int/mediacentre/factsheets/(last accessed 10 August 2016)

3 World Health Organization. Fact sheet: Obesity and overweight, June 2016. http://www.who.int/mediacentre/factsheets/ (last accessed 10 August 2016).

4 Schmitt-Egenolf M. PsoReg - the Swedish registry for systemic psoriasis treatment. The registry's design and objectives. Dermatology 2007; 214:112-17.

5 McPhail S, Schippers M. An evolving perspective on physical activity counselling by medical professionals. BMC Family Practice 2012; 13:31.

6 Keyworth C, Nelson PA, Chew-Graham CA et al. Communicating cardiovascular disease risk to people with psoriasis: what techniques do practitioners use? Int J Behav Med 2016; 23:168-178.

7 Torres T, Alexandre J, Mendonça D et al. Levels of physical activity in patients with severe psoriasis: a cross-sectional questionnaire study. Am J Clin Dermatol 2014; 15:129-135.

8 Do YK, Lakhani N, Malhotra R et al. Association between psoriasis and leisure-time physical activity: findings from the National Health and Nutrition Examination Survey. J Dermatol 2015; 42:148-153.

9 Koo J. Population-based epidemiologic study of psoriasis with emphasis on quality of life assessment. Dermatol Clin 1996; 14:485496.

10 Frankel HC, Han J, Li T et al. The association between physical activity and the risk of incident psoriasis. Arch Dermatol 2012; 148:918-924.

11 Naldi L, Conti A, Cazzaniga S et al. Diet and physical exercise in psoriasis: a randomized controlled trial. Br J Dermatol 2014; 170:634-642. 